\title{
Photocatalytic Segmented Nanowires and Single-step Iron Oxide Nanotube Synthesis: Templated Electrodeposition as all-round Tool
}

\author{
Michiel G. Maas ${ }^{1}$, Eddy J.B. Rodijk ${ }^{1}$, Wouter Maijenburg ${ }^{1}$, Johan E. ten Elshof ${ }^{1}$, Dave H.A. \\ Blank $^{1}$ \\ ${ }^{1} \mathrm{MESA}^{+}$Institute for Nanotechnology, University of Twente, P.O. Box 217, Enschede, NL- \\ 7500AE, The Netherlands
}

\begin{abstract}
Templated electrodeposition was used to synthesize silver-zinc oxide nanowires and iron oxide $\left(\mathrm{Fe}_{2} \mathrm{O}_{3}\right)$ nanotubes in polycarbonate track etched (PCTE) membranes. Metal/oxide segmented nanowires were made to produce hydrogen gas from a water/methanol mixture under ultraviolet irradiation. It was observed that gas production increased during irradiation. Iron oxide nanotubes were synthesized via a gel synthesis route, avoiding clogging of the membrane pores during growth. The nanotubes formed without thermal after-treatment. Transmission electron microscopy (TEM) analysis and selected area electron diffraction (SAED) revealed a completely amorphous iron oxide structure. By demonstrating the synthesis of photocatalytically active segmented nanowire and nanotubes without post-treatment steps, templated electrodeposition can be a versatile and low cost tool for nanowires with designed functionality or fast nanotube synthesis.
\end{abstract}

\section{INTRODUCTION}

One-dimensional nanostructures -such as nanowires and nanotubes- have attracted much attention over the years. Their unique physical and chemical properties make them very promising building blocks for future applications, such as nanosensors [1-3] measuring molecules or gases in nanomolars or less, optical or molecular tags [4-6] for cell tracking applications, and even self-propelling nanomotors [7-9] which, for example, can be used as nanocarriers for molecules or medicines. Each application can utilize a specific property of a nanowire or nanotube, whether it has been made of metal or oxide. However, nanowires and nanotubes will only be suitable as building blocks on the nanoscale if their quality, e.g. size distribution, crystallinity and mechanical strength, are within certain limits. Commercial implementation demands a reliable, simple and low cost technique to produce (segmented) nanowires or nanotubes. Ideally, synthesis of nanowires and nanotubes is combined in a single technique without changing steps in the process. We consider templated electrodeposition to be a viable option for this.

In this contribution, we report the growth of segmented nanowires in polycarbonate track-etched (PCTE) membrane pores from a suitable electrolyte solution. Applying an overpotential initiated nanowire growth. Segments could be made of a variety of materials, for example silver and zinc oxide. The combination of silver and zinc oxide in a single nanowire enabled the production of hydrogen from a water/methanol solution under the influence of ultraviolet radiation. Nanotube formation is demonstrated by a deposition strategy that involves the formation of an iron hydroxide gel and a drying step to yield iron oxide $\left(\mathrm{Fe}_{2} \mathrm{O}_{3}\right)$ nanotubes, but no thermal treatment. 


\section{EXPERIMENTAL DETAILS}

Nuclepore ${ }^{\mathrm{TM}}$ polycarbonate track-etched (PCTE) membranes of 6 micrometer thickness and pore diameters of 50 and 200 nanometers were purchased from Whatman Inc. Membrane pore density is between $1 \times 10^{5}-6 \times 10^{8}$ pores $/ \mathrm{cm}^{2}$. Prior to deposition, a gold layer with a thickness of $\sim 50 \mathrm{~nm}$ was sputtered on one side of the membrane. After sputtering the gold coated side of the membrane was attached to a glass slide with double-sided tape.

The membrane/glass combination was attached as working electrode in a 3-electrode setup using a Bank Elektronik POS 73 potentiostat. As counter electrode a small piece of platinum mesh was used. The reference potential was set by a $3 \mathrm{M} \mathrm{KCl} \mathrm{Ag/AgCl} \mathrm{reference} \mathrm{electrode} \mathrm{(REF} \mathrm{321,}$ Radio Analytical).

Silver segments were deposited from an electrolyte containing $0.20 \mathrm{M} \mathrm{AgNO} 3(99+\%$, Acros) and $0.10 \mathrm{M} \mathrm{H}_{3} \mathrm{BO}_{3}$ (99.99\%, Sigma-Aldrich). The $\mathrm{pH}$ was adjusted to 1.5 with nitric acid. Deposition occurred at $+0.10 \mathrm{~V}$. Zinc oxide segments were deposited from an electrolyte containing $0.10 \mathrm{M}$ $\mathrm{Zn}\left(\mathrm{NO}_{3}\right)_{2} \cdot 6 \mathrm{H}_{2} \mathrm{O}\left(98 \%\right.$, Sigma-Aldrich) at $70^{\circ} \mathrm{C}$ at $-1.00 \mathrm{~V}$.

The iron hydroxide gel was made using an electrolyte containing $0.02 \mathrm{M} \mathrm{Fe}\left(\mathrm{NO}_{3}\right)_{3} \bullet 9 \mathrm{H}_{2} \mathrm{O}(98+\%$, Sigma-Aldrich), 0.430M HNO 3 (65\%, Acros) and $0.425 \mathrm{M} \mathrm{NaOH}$ (pellets, Sigma-Aldrich). Gel formation occurred at $-1.00 \mathrm{~V}$.

After deposition, the PCTE membranes were dissolved in dichloromethane (Merck). To isolate wires/tubes on a substrate for analysis, a droplet of dichloromethane containing wires/tubes and dissolved polycarbonate was placed on the substrate and dried. After drying, the substrate was rinsed with fresh dichloromethane by holding it for $15 \mathrm{~s}$ in the solvent.

For the gas measurements the dichloromethane/membrane/wire solution was centrifuged and decanted and rinsed three times with fresh dichloromethane before adding them to a water/methanol solution.

Analysis of segmented nanowires was performed with a Zeiss HR-LEO 1550 FEF scanning electron microscope (SEM). Nanotubes were characterized by an analytical TEM from FEI instruments.

\section{DISCUSSION}

\section{Segmented nanowires}

Formation of hydrogen by photocatalysis at room temperature has been reported in literature [1012]. Usually titanium oxide is used to create an electron-hole pair under UV irradiation to split water or methanol into hydrogen and oxygen. Titanium oxide is usually combined with a metal from the platinum group to increase the efficiency of the process by preventing quick recombination of an electron and a hole $[13,14]$. To our knowledge, demonstrations of water splitting under UV-irradiation have been performed only by macroscale electrodes. Here, hydrogen production by electrodeposited silver and zinc oxide segmented nanowires is pursued as an alternative to a titanium oxide/platinum group metal combination, see figure 1 . 


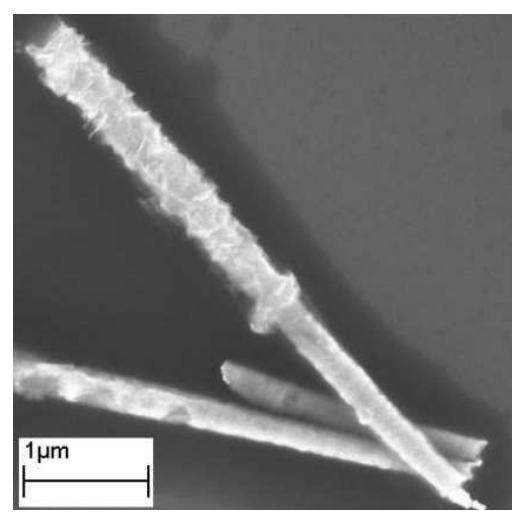

Figure 1. Silver-zinc oxide nanowire. The rougher surface is the zinc oxide segment; the smoother surface is the silver segment. The dark areas in the figure are polycarbonate residues.

A mixture of water and methanol (1:4 ratio) was used to demonstrate hydrogen formation [14]. Because the electrodeposited zinc oxide is an n-type semiconductor with a band gap of $3.25 \mathrm{eV}$, an electron-hole pair can be created by UV irradiation. Silver is used as the metallic part of the wire to prevent a quick recombination of the electron-hole pair. In addition, the water/methanol mixture functions as a hole scavenger to prevent quick recombination of an electron-hole pair more efficiently [14].

The overall reaction mechanism of hydrogen formation is shown in reaction 1.

$\mathrm{CH}_{3} \mathrm{OH}(\mathrm{l})+\mathrm{H}_{2} \mathrm{O}(\mathrm{l})=\mathrm{CO}_{2}(\mathrm{~g})+3 \mathrm{H}_{2}(\mathrm{~g})$

To produce hydrogen, a large quantity of segmented silver-zinc oxide wires was put in a quartz tube, filled with methanol/water, and irradiated with a commercially available UV source. Visual observation clearly showed that small bubbles formed on the bottom of the quartz tube and rose through the solvent. Upon exposure to UV light, the rate of bubble formation increased. Figure 2 shows the number of gas bubbles per unit time upon UV-irradiation.

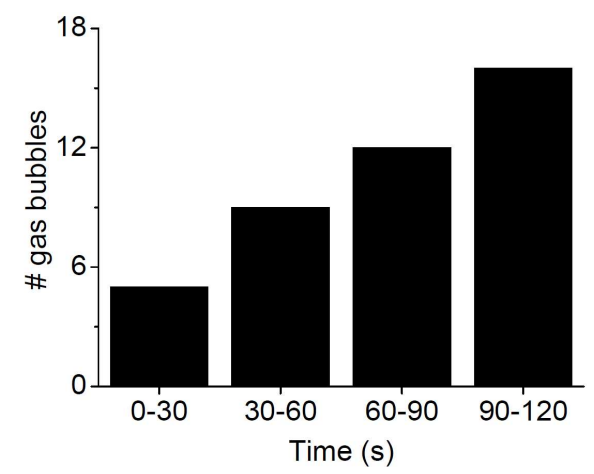

Figure 2. Counted number of rising gas bubbles to the surface per time interval. At the beginning of each interval, counting was restarted.

\section{Iron oxide nanotubes}

Nanotubes formed during the drying of an electrochemically induced iron hydroxide gel inside polycarbonate track etched (PCTE) membranes. Nucleation during the electrochemical reaction 
was suppressed $[15,16]$. By maintaining a high $\mathrm{pH}$ and relative low temperature (RT) during gel formation, iron hydroxide species did not redissolve or nucleate.

The high $\mathrm{pH}$ was maintained by reducing nitrate species continuously at the working electrode, following reduction reaction 2 [17]. At the start of the process, this reaction generates a high $\mathrm{pH}$ locally. The formed hydroxyls diffuse through the pore, creating a diffusion zone and front.

$\mathrm{Fe}(\mathrm{III})$ ions that were already present in the membrane pores enters the diffusion zone and reacts with the hydroxide ions (reaction 3 ).

Due to the fact that a high $\mathrm{pH}$ was maintained inside the diffusion zone and gel formation took place at room temperature, formation of iron oxide (reaction 4) was suppressed. Only after removal from the electrolyte solution, this reaction was initiated by the release of water during drying.

$$
\begin{aligned}
& \mathrm{NO}_{3}^{-}+\mathrm{H}_{2} \mathrm{O}+2 \mathrm{e}^{-}=\mathrm{NO}_{2}^{-}+2 \mathrm{OH}^{-} \\
& \mathrm{Fe}^{3+}+3 \mathrm{OH}^{-}=\mathrm{Fe}(\mathrm{OH})_{3} \\
& 2 \mathrm{Fe}(\mathrm{OH})_{3}=\mathrm{Fe}_{2} \mathrm{O}_{3}+3 \mathrm{H}_{2} \mathrm{O}
\end{aligned}
$$

Figure 3 shows a chronoamperogram of the gel formation process during 20 minutes. Period I shows the charging of the double layer capacitance with time. Period II indicates a current increase, which suggests an increasing electrode surface by nucleation and growth processes $[15,16]$ or an increasing nitrate reduction rate. Because the process is carried out at low temperatures, nucleation is not favored. We interpret the measured current increase as a build-up process of the hydroxide diffusion zone and front, thereby suppressing nucleation at the electrode surface. After the diffusion zone and front have been created, the process entered period III. The interior of the pore filled with gel, which influenced the speed of transportation of nitrates from the pore mouth towards the electrode at the end of the pore. As a result the current dropped slowly but steadily because of decreasing diffusion rates of nitrate species through the pores towards the electrode.

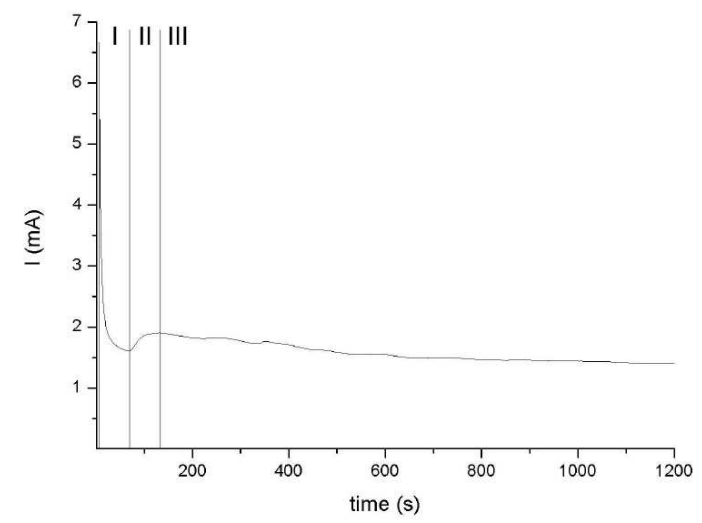

Figure 3. Chronoamperogram of iron hydroxide gel formation. Three periods can be distinguished. I) double layer capacitance charging; II) build-up of local $\mathrm{pH}$ zone and iron hydroxide formation; III) simultaneous diffusion of reactants towards electrode and spreading of iron hydroxide gel in PCTE membrane pores. 
After hydroxide formation was stopped, the membrane was removed from the electrolyte, dried in air and dissolved in dichloromethane. The dichloromethane/nanotube solution was dispersed on a carbon-coated copper TEM grid. Dichloromethane was evaporated and the TEM grid was rinsed with fresh dichloromethane to remove any polycarbonate contaminations. Figure 2 shows the TEM analysis and a selected area electron diffraction (SAED) pattern in the middle.

Nanotubes of up to 3.5 micrometer in length were formed during a 20 minute synthesis. SAED shows a completely amorphous material. This indicates that no nucleation occurred during iron hydroxide formation or during drying. Furthermore, on the right a more complex X-shaped tubular structure is shown. Note that the crossing is a completely tubular structure and cannot be ascertained without the gel formation step. We believe that this technique would also enable the formation of more complex nanotubular structures, which is especially helpful for fluidics in nanochannels. Figure 4 shows a few tubular structures that have not fully opened during drying. Instead, a low angle $\mathrm{V}$-shape interface is observed. We believe that this is a result of the drying process as schematically depicted in Figure 5. A meniscus formed during drying and progressed through the pore in time, thereby creating the nanotubes. It was estimated that 70 percent of the structures measured during TEM analysis were intact nanotubes.
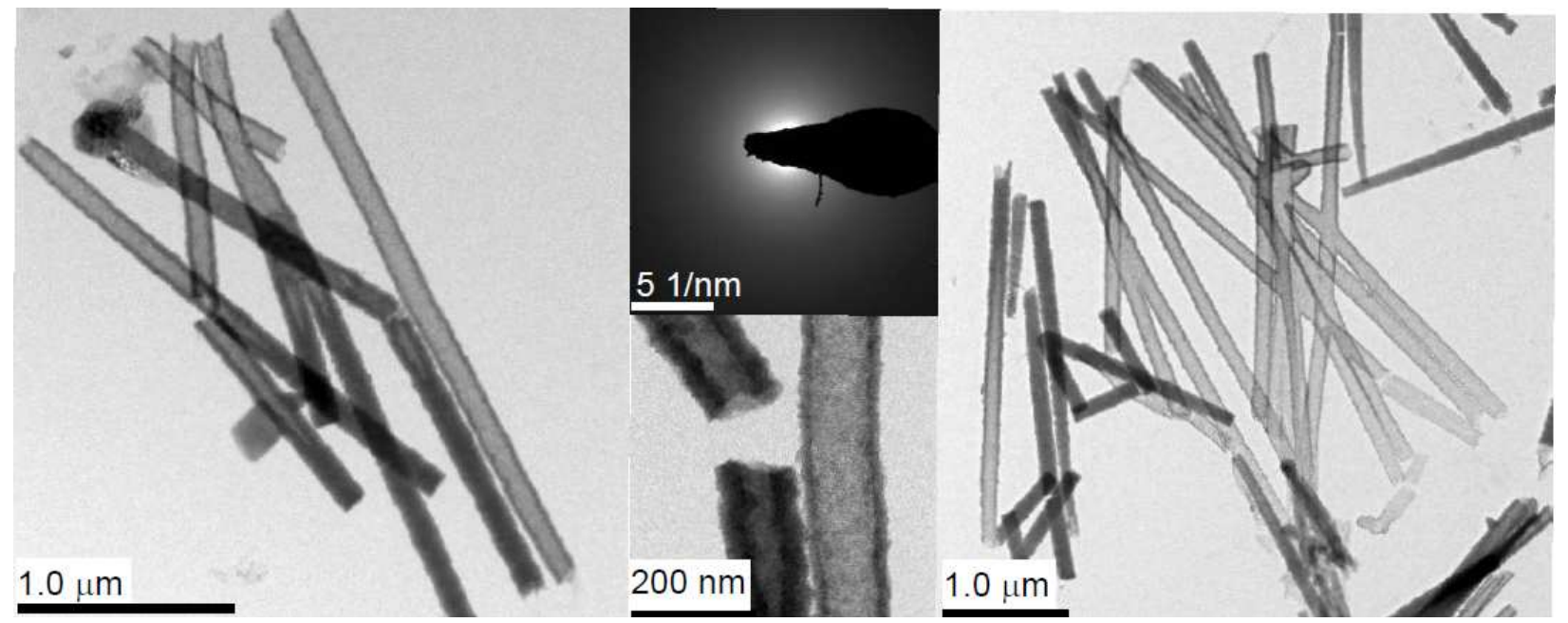

Figure 4. TEM analysis of iron oxide nanotubes. Left) a $\sim 3$ micrometer nanotube is shown.

Nanotubular structures with a low angle V-shaped interface are also shown as an example of the drying process; Middle) The area selected for the electron diffraction and its pattern (bottom and top, respectively); Right) $\mathrm{X}$-shaped nanotubular structures resulting from crossing PCTE membrane pores.
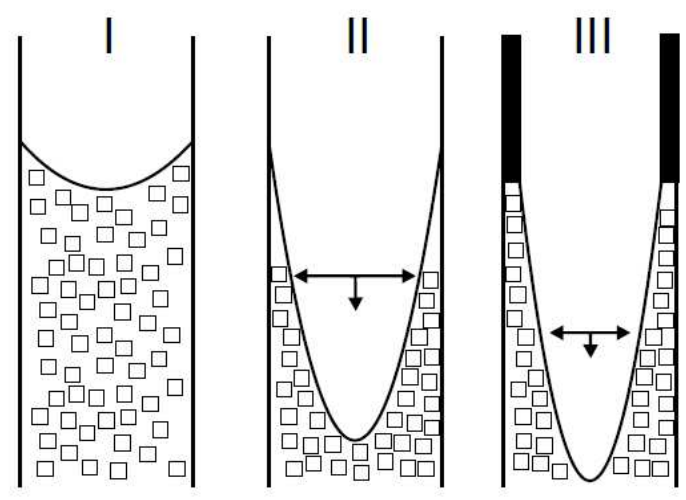
Figure 5. Schematic illustration of nanotube formation during drying. I) meniscus at the start of the drying process; II) progression of the meniscus; III) tube formation. Gel is pushed aside and dries forming amorphous iron oxide nanotubes.

\section{CONCLUSIONS}

Silver-zinc oxide segmented nanowires were synthesized by electrodeposition by changing the electrolyte solution during growth. They can offer an alternative to titanium dioxide-platinum based photocatalytic hydrogen formation. Amorphous iron oxide nanotubes were synthesized without special pre-treatment of a polycarbonate track-etched membrane nor by heat treatment after synthesis. By controlling the electrolyte composition, the synthesis of nanowires and nanotubes is feasible with the same setup and settings, thus keeping costs as low as possible.

\section{ACKNOWLEDGMENTS}

Financial support by the Dutch Ministry of Economic Affairs in the framework of the NanoNed program is acknowledged.

\section{REFERENCES}

1. Y.W. Heo, D.P. Norton, L.C. Tien, Y. Kwon, B.S. Kang, F. Ren, S.J. Pearton, J.R. LaRoche, Mat. Sci. Eng. R, 47, 1 (2004).

2. $\quad$ R. Fan, R. Karnik, M. Yue, D. Li, A. Majumdar, P. Yang, Nano Lett. 5 (9), 1633 (2005).

3. F. Patolsky, G. Zheng, C.M. Lieber, Anal. Chem. 78 (13), 4260 (2006).

4. C.D. Keating, M.J. Natan, Adv. Mater. 15 (5), 451 (2003).

5. $\quad$ L.A. Bauer, D.H. Reich, G.J. Meyer, Langmuir 19, 7043 (2003).

6. J. Wang, J. Mater. Chem. 18, 4017 (2008).

7. W.F. Paxton, S. Sundararajan, T.E. Mallouk, A. Sen, Angew. Chem. Int. Ed. 45, 5420 (2006).

8. Y. Wang, R.M. Hernandez, D.J. Bartlett, J.M. Bingham, T.R. Kline, A. Sen, T.E. Mallouk, Langmuir 22 (25), 10451 (2006).

9. J. Wang, ACS Nano 3 (1), 4 (2009).

10. A. Fujishima, K. Honda, Nature 238, 37 (1972).

11. A. Fujishima, K. Kohayakawa, K. Honda, J. Electrochem. Soc. 122, 1487 (1975).

12. J. Nowotny, T. Bak, M.K. Nowotny, L.R. Sheppard, Int. J. Hydrogen Energ. 32, 2609 (2007).

13. H. Dannetun, I. Lundstroem, L.G. Petersson, J. Appl. Phys. 70, 453 (1991).

14. W.G. Lin, W.D. Yang, I.L. Huang, Energ. Fuel. 23, 2192 (2009).

15. H. Bort, K. Juettner, W.J. Lorenz, G. Staikov, E. Budevski, Electrochim. Acta 28 (7), 985 (1983).

16. A. Radisic, P.M. Vereecken, J.B. Hannon, P.C. Searson, F.M. Ross, Nano Lett. 6 (2), 238 (2006).

17. G.H.A. Therese, P.V. Kamath, Chem. Mater. 12 (5), 1195 (2000). 